\title{
A patient with recurrent palpitations and unusual anatomy
}

\author{
C. J. M. Lawson · A. D. Margulescu · J. Barry
}

Published online: 19 March 2020

(c) The Author(s) 2020

\section{Answer}

Complete atresia of the inferior vena cava (IVC) with cavo-azygos (CA) continuity was diagnosed by contrast venography performed using a long sheath (SL0, Abbott Medical, USA) (Fig. 1a). Following the CA vein route, a decapolar and a quadripolar catheter were advanced into the superior vena cava, right atrium, and then into the coronary sinus and right ventricle, respectively (Fig. 1b). However, the right subclavian vein needed to be used to map the triangle of Koch with the ablation catheter, due to better reach and stability at this region compared with the CA route (Fig. 1c).

IVC atresia with CA continuity is a rare congenital anomaly that results from lack of interruption of the right cardinal vein at the level of the diaphragm during embryological development [1]. As a result, the intrahepatic trajectory of the IVC is not formed, and the hepatic veins will drain separately into the right atrium. Fig. 1d shows a schematic representation of normal venous anatomy vs. CA continuity. CA continuity may be associated with more extensive embryological abnormalities, such as the heterotaxy syndrome (abnormal arrangement of internal organs across the left-right axis of the body) [2]. In our patient, chest X-ray, abdominal ultrasound and echocardiogram revealed normal internal organ arrangement.

For electrophysiology procedures, IVC atresia with CA continuity can cause significant challenges, especially if left atrial access is required, because the impossibility of performing transseptal puncture through the usual inferior approach [3, 4]. How-

C. J. M. Lawson · A. D. Margulescu · J. Barry ( $₫)$ Department of Cardiology, Morriston Regional Cardiac Centre, Swansea, SA6 6NL, UK james.barry@wales.nhs.uk ever, right-sided ablations (including ablation of atrioventricular nodal re-entrant tachycardia) can be performed with small variations of standard techniques, by looping the catheters back into the right cardiac chambers through the superior vena cava, as demonstrated in this case.

Conflict of interest C.J.M. Lawson, A.D. Margulescu and J. Barry declare that they have no competing interests.

Open Access This article is licensed under a Creative Commons Attribution 4.0 International License, which permits use, sharing, adaptation, distribution and reproduction in any medium or format, as long as you give appropriate credit to the original author(s) and the source, provide a link to the Creative Commons licence, and indicate if changes were made. The images or other third party material in this article are included in the article's Creative Commons licence, unless indicated otherwise in a credit line to the material. If material is not included in the article's Creative Commons licence and your intended use is not permitted by statutory regulation or exceeds the permitted use, you will need to obtain permission directly from the copyright holder. To view a copy of this licence, visit http://creativecommons.org/licenses/by/4.0/.

\section{References}

1. Hikspoors JPJM, Soffers JHM, Mekonen HK, et al. Development of the human infrahepatic inferior caval and azygos venous systems. JAnat. 2015;226:113-25.

2. Kim SJ. Heterotaxy syndrome. Korean Circ J. 2011;41:22732.

3. Okajima K, Nakanishi T, Ichibori H, et al. Trans-aortic pulmonaryvein isolation usingmagnetic navigation systemfor paroxysmal atrial fibrillation in a patient with dextrocardia, situs inversus, and inferior vena cava continuity with azygos vein. JArrhythm. 2018;34:583-5.

4. Kato H, Kubota S, Goto T, et al. Transseptal puncture and catheter ablation via the superior vena cava approach for persistent atrial fibrillation in a patient with polysplenia syndrome and interruption of the inferior vena cava: contact force-guided pulmonary vein isolation. Europace. 2017;19:1227-32. 
Fig. 1 Inferior vena cava atresia with cavo-azygos (CA) continuity accounts for the unusual placement of the electrophysiology catheters from the right inguinal region into the heart. a LAO 30, left anterior oblique 30 degrees view; b RAO 30, right anterior oblique 30 degrees view; c Contrast venogram helped deliniate the anatomical structures through which the electrophysiology catheter and sheaths were advanced from the right inguinal region; d Schematic representation of normal venous anatomy (left) vs. CA continuity (right). CS coronary sinus, $R A$ right atrium $R V$ right ventricle $S P$ slow pathway $T V$ tricuspid valve, SVC superior vena cava, $I V$ innominate vein, $A V$ azygos vein, $H A V$ hemiazygos vein, $H V$ hepatic veins, $R R V$ right renal vein, $L R V$ left renal vein
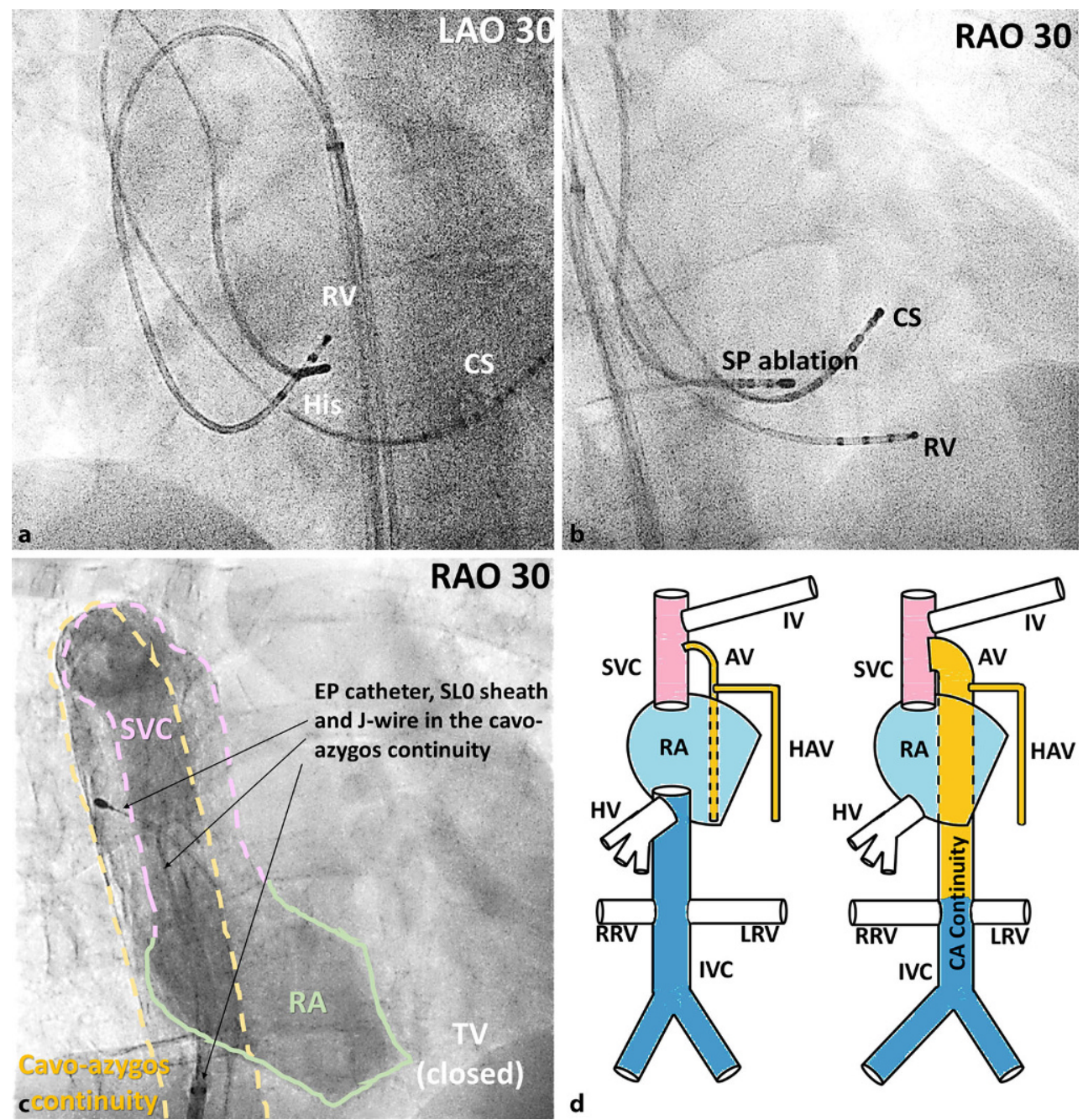